The impact of drug characteristics on medication adherence has not been studied across disease states. The purpose of this study was to describe medication adherence by drug characteristics across multiple disease states to identify drug-specific factors that may serve as targets for future intervention to improve medication adherence. Methods: We conducted a retrospective analysis of patients with one diagnosis and oral medication using medical claims and pharmacy utilization data for the following conditions: asthma/COPD, cancer, depression, diabetes, hypercholesterolemia, hypertension, multiple sclerosis (MS) or osteoporosis between January 1, 2007 and March 31, 2009. Adherence was calculated using the Medication Possession Ratio for each patient from prescription fills occurring within a 12-month window. Results: Analysis of members with one diagnosis and one medication $(n=14,875)$ found the majority female $(61 \% ; n=9059)$ and Caucasian $(83 \% ; n=12,317)$. The most commonly occurring conditions were hypertension $(n=5,505)$, depression $(n=4,349)$ and hyperlipidemia $(n=2,744)$. Generic utilization rates varied widely by diagnosis as did copayment. Binary adherence $(>80 \%)$ varied significantly by diagnosis, drug class (within condition), generic use, and copayment. Regression analysis showed significant adherence variations within condition for brand and generic use and copayment. Discussion: We ascertained adherence varies by disease state, drug class within disease states, and drug characteristics. Next steps include an interventional study that will use this data to target specific populations by drug-related characteristics.

Keywords: Medication Adherence; Medication Compliance; Chronic Illness and Multimorbidity

doi:10.3121/cmr.2012.1100.cc3-03

\section{Comparative Effectiveness Research}

PS1-33:

Cancer Screening Guideline Development, Implementation and Dissemination: Connection Points for Researchers and Health Care Delivery Systems for Comparative Effectiveness Research

Diana Buist ${ }^{1}$; Leah Tuzzio $^{1}$; Robert Greenlee ${ }^{2}$; Terry Field $^{3}$; Andrew Williams ${ }^{4}$; Sheila Weinmann ${ }^{5}$; Natasha Stout ${ }^{6}$; Aruna Kamineni ${ }^{1}$; Chyke Doubeni ${ }^{3}$

${ }^{1}$ Group Health Cooperative; ${ }^{2}$ Marshfield Clinic; ${ }^{3}$ Meyers Primary Care Institute/University of Massachusetts Medical School; ${ }^{4}$ Kaiser Permanente Hawaii; ${ }^{5}$ Kaiser Permanente Northwest; ${ }^{6}$ Harvard Pilgrim Health Care Institute, Harvard Medical School

Background/Aims: Cancer Screening Effectiveness And Research in Community-based Healthcare (SEARCH) involves 8 Cancer Research Network (CRN) sites and includes two comparative effectiveness research (CER) proof-of-principle studies anchored in colorectal and cervical cancer screening. This portion of the project was designed to: 1) understand how cancer screening guidelines are developed, implemented and disseminated at each site; 2) identify the appropriate clinical leads within each health plan to further engage in bidirectional discussions about future comparative effectiveness cancer screening research and to help disseminate SEARCH findings across the CRN sites; and 3) identify opportunities for researchers and clinical leaders to collaborate on informing each other and/or conducting comparative effectiveness research together. Methods: We conducted 18 semi-structured interviews with clinical leaders across 8 health plans (GHC, Geisinger, Marshfield, Fallon, Harvard Pilgrim, Kaiser: Northwest, Hawaii, Northern California) and with one State-wide quality improvement group. Results: There is considerable site variability in guideline development, implementation and dissemination processes. Much work goes into reviewing evidence and developing clinical guidelines; parallel work and evidence reviews happen across. Many different vehicles are used for guideline dissemination; opportunities exist to study how to most effectively disseminate guidelines to patients and providers to yield optimal screening. Clinical leaders provided critical information around principles and required elements for conducting CER. Opportunities, barriers and principles around conducting CER will be presented. Discussion: There are a number of areas that can be immediately leveraged: to further facilitate learning health care systems, and for researchers and delivery systems to improve the cancer screening guideline development, dissemination and implementation. We see important opportunities for helping the research network and its member sites develop and use guidelines more efficiently to improve care and enhance the research we conduct. Research teams must work with key leaders to identify high priority CER studies and to hard-wire the conduct of CER trials into standard clinical work-flow.

Keywords: Stakeholder Engagement; Guideline Development; Comparative Effectiveness Research

doi:10.3121/cmr.2012.1100.ps1-33

PS1-34:

Overcoming Barriers for Tumor Block Retrieval and Testing in a Multisite Cancer Research Network Project

Tia Kauffman'; Yeehwa Daida ${ }^{2}$; Michelle Groesbeck ${ }^{3}$; Terrie Kitchner ${ }^{5}$; Paul Meier $^{3}$; Brian Owens ${ }^{4}$; Denise Schwarzkopf ${ }^{1}$; Alanna Kulchak Rahm ${ }^{6}$

${ }^{1}$ Kaiser Permanente Northwest; ${ }^{2}$ Kaiser Permanente Hawaii; ${ }^{3}$ Henry Ford Health System; ${ }^{4}$ Marshfield Clinic; ${ }^{5}$ HealthPartners; ${ }^{6}$ Kaiser Permanente Colorado

Background/Aims: KRAS testing is a pharmacogenomic test to help decide how to treat patients with metastatic colorectal cancer (mCRC). The CERGEN (Comparative Effectiveness Research in Genomics \& Personalized Medicine for Colorectal Cancer) project, a collaboration among eight institutions of the NCI-funded Cancer Research Network (CRN) and four academic partners, utilized KRAS test results to evaluate overall survival in mCRC patients. As a part of this project, we needed to retrospectively test all patients that had not had the testing done as part of their clinical care. This project is a large, multi- site CRN project utilizing archived tumor tissue. Methods: For cases that had not been previously tested for KRAS mutation status at the seven data collection sites, we tested archived pathology specimens from the primary tumor. Testing was planned to be performed at one central laboratory that was not affiliated with any of the institutions. Each site needed to identify study patients that had not been KRAS tested, retrieve blocks that could be utilized for research, section blocks per laboratory requirements, and return testing results to the lead site. Results: All sites required varying degrees of approvals, paperwork, and sample processing. Despite many challenges, we successfully performed KRAS testing on tumor tissue from over 400 blocks. This poster will present the issues and challenges encountered at each of the seven sites in performing testing on tumor blocks, including: varying IRB requirements at each site; challenges around IRB ceding; site-specific biospecimen committees; timing around slides sent to the laboratory; staffing challenges in pathology; and varying material transfer and data sharing agreements. Additionally, the pathology department at one site required in-house testing only. We will also share our successes including: pilot testing the process before implementation; shipping blocks to another site for sectioning when there were pathology staffing shortages; working to standardize IRB applications; and working with the laboratory to ensure quality control in all of the samples. Discussion: We were successfully able to standardize tumor block testing for research across seven HMORN sites. We hope that our "lessons learned" are helpful for other HMORN projects that wish to use archived tissue.

Keywords: Genetics; Tumor blocks; Comparative Effectiveness Research doi:10.3121/cmr.2012.1100.ps1-34

CC4-01:

CER-HUB: An Informatics Platform for Conducting Comparative Effectiveness Research with Comprehensive Electronic Medical Record Data

Brian Hazlehurst ${ }^{1}$; Victor Stevens ${ }^{1}$; Richard Mularski ${ }^{1}$; Mary Ann McBurnie ${ }^{1}$

${ }^{1}$ Kaiser Permanente Northwest

Background/Aims: The American Recovery and Reinvestment Act (ARRA) provided \$1.1 billion for Comparative Effectiveness Research (CER) with the goal of generating new evidence on the effectiveness of different treatments, diagnostic procedures, and care models under "real world" conditions. AHRQ awarded $\$ 100$ million of ARRA funds to a dozen R01 projects to develop clinical infrastructure focusing on use of comprehensive electronic medical record (EMR) systems for large-scale, multi-institutional CER. However, numerous informatics challenges must be overcome when 